\title{
INVARIANT SUBSPACES OF THE DIRICHLET SHIFT AND PSEUDOCONTINUATIONS
}

\author{
STEFAN RICHTER AND CARL SUNDBERG
}

\begin{abstract}
In this paper we study extremal functions for invariant subspaces of the Dirichlet shift, i.e., solutions $\varphi$ of the extremal problem$$
\sup \left\{\left|f^{(n)}(0)\right| /\|f\|_{D}: f \in \mathscr{M}, f \neq 0\right\} .
$$

Here $n$ is the smallest nonnegative integer such that the sup is positive. It is known that such a function $\varphi$ generates $\mathscr{M}$. We show that the derivative $(z \varphi)^{\prime}$ has a pseudocontinuation to the exterior disc. This pseudocontinuation is an analytic continuation exactly near those points of the unit circle where $\varphi$ is bounded away from zero. We also show that the radial limit of the absolute value of an extremal function exists at every point of the unit circle. Some of our results are valid for all functions that are orthogonal to a nonzero invariant subspace.
\end{abstract}

\section{INTRODUCTION}

The Dirichlet space $D$ is the space of all analytic functions $f(z)=\sum \hat{f}(n) z^{n}$ on the open unit disc $\mathbb{D}$ which have a finite Dirichlet integral

$$
D(f)=\iint_{D}\left|f^{\prime}(z)\right|^{2} d A(z)
$$

here $d A(z)=(1 / \pi) r d r d t$ denotes normalized area measure on $\mathbb{D}$. A norm on $D$ is defined by

$$
\|f\|_{D}^{2}=\|f\|_{H^{2}}^{2}+D(f)=\sum_{n=0}^{\infty}(n+1)|\hat{f}(n)|^{2} .
$$

The Dirichlet shift $\left(M_{z}, D\right)$ is the operator of multiplication by $z$ on $D$. The lattice of all (closed) invariant subspaces of $\left(M_{z}, D\right)$ will be denoted by $\operatorname{Lat}\left(M_{z}, D\right)$. Let $\mathscr{M} \in \operatorname{Lat}\left(M_{z}, D\right), \mathscr{M} \neq(0)$ and let $n$ be the smallest nonnegative integer such that there are functions $f$ in $\mathscr{M}$ whose $n$th Taylor coefficient $\hat{f}(n)$ is nonzero. We say that a function $\varphi \in \mathscr{M}, \varphi \neq 0$, is extremal for $\mathscr{M}$ if

$$
|\hat{\varphi}(n)| /\|\varphi\|_{D}=\gamma(\mathscr{M})=\sup \left\{|\hat{f}(n)|: f \in \mathscr{M},\|f\|_{D}=1\right\} .
$$

Received by the editors August 19, 1991 and, in revised form, January 17, 1992.

1991 Mathematics Subject Classification. Primary 47B38, 47A15, 46E20; Secondary 31A20, $30 \mathrm{H} 05$.

Key words and phrases. Dirichlet space, invariant subspaces, pseudocontinuation, analytic continuation.

Research supported by the National Science Foundation and the Tennessee Science Alliance. 
It is known that $\operatorname{dim} \mathscr{M} \ominus z \mathscr{M}=1$ for every nonzero invariant subspace (see [15, Theorem 2]), and it is thus easy to check that a function $\varphi \in \mathscr{M}$, $\varphi \neq 0$, is extremal if and only if $\varphi \in \mathscr{M} \ominus z \mathscr{M}$. This means that extremal functions always exist and they are unique up to constant multiples. Yet another way of thinking about extremal functions arises through the reproducing kernel functions. For an integer $n \geq 0$ the function $k_{n}(z)=z^{n} /(n+1)$ is the reproducing kernel for the $n$th Taylor coefficient at 0 , i.e., $k_{n}$ is the unique function that satisfies $\hat{f}(n)=\left(f, k_{n}\right)_{D}$ for every $f \in D$. If we let $P$ denote the projection of $D$ onto the invariant subspace $\mathscr{M}$, then $P k_{n}$ is an extremal function for $\mathscr{M}$ if $P k_{n} \neq 0$ and $P k_{0}=\cdots=P k_{n-1}=0$.

Extremal functions play an important role in the theory of invariant subspaces of spaces of analytic functions. For example, according to Beurling's theorem every invariant subspace of $H^{2}$ is generated by an inner function, and the inner functions are just the normalized extremal functions in $H^{2}$. For the Bergman space $L_{a}^{2}(\mathbb{D})$ a result of $\mathrm{H}$. Hedenmalm states that normalized extremal functions are expansive multipliers (see [9] for the details), and this has interesting consequences for the factorization of functions in $L_{a}^{2}(\mathbb{D})$.

In the context of the Dirichlet space extremal functions were considered by L. Carleson in his investigations of sets of uniqueness for $D$ [2]. His result can be summarized as follows: With certain subsets $E$ of the unit circle $\mathbb{T}$ (so-called Carleson thin sets), he associates the invariant subspace $D_{E}$ of all functions whose radial limit vanishes on $E$ except for a set of logarithmic capacity zero. Carleson proves that the derivative of an extremal function for $D_{E}$ has an analytic continuation to $\mathbb{C} \backslash E[2$, Theorem 7]. A part of our Lemma 3.1 (a) is a generalization of this result to arbitrary invariant subspaces. We note that Carleson uses a different norm on $D$ than we do, so his extremal functions differ slightly from ours.

Shapiro and Shields use extremal functions and estimates on the quantity $\gamma(\mathscr{M})$ of $(1.1)$ to get conditions for when a set $\left\{z_{n}\right\} \subseteq \mathbb{D}$ may be the zero sequence of a function in $D$ [19]. They also apply their method to other spaces of analytic functions.

Our current interest in extremal functions in the Dirichlet space comes from the fact that one has a Beurling-type theorem for $D$. Every invariant subspace $\mathscr{M}$ of $D$ is generated by an extremal function. More precisely $\mathscr{M}=\varphi D\left(m_{\varphi}\right)$, where $\varphi$ is a normalized extremal function, $m_{\varphi}$ is a certain absolutely continuous measure on $\mathbb{T}$, and $D\left(m_{\varphi}\right)$ is a Dirichlet-type space associated with $m_{\varphi}$ (see [14, Theorem 7.1]). Up to this point our only general knowledge about extremal functions is contained in Theorem 3.1 of [17]: extremal functions in $D$ are multipliers of the Dirichlet space, i.e., functions $\varphi$ such that $\varphi D \subseteq D$.

Note that extremal functions are contained in the invariant subspace $\mathscr{M}$ and also in the orthocomplement $(z \mathscr{M})^{\perp}$. As mentioned above $\mathscr{M}$ is generated by an extremal function $\varphi$, and an analogous statement is true for $(z \mathscr{M})^{\perp}$, namely $(z \mathscr{M})^{\perp}$ is the smallest invariant subspace of $M_{z}^{*}$ which contains $\varphi$ (see $[13$, p. 215]). Thus one expects the extremal functions to reflect the analytical properties of the functions in both $\mathscr{M}$ and $\mathscr{M}^{\perp}$.

In $\S \S 2$ and 3 below we investigate general functions in $\mathscr{M}^{\perp}$. Before we state the results we need to state and recall some definitions. $\mathbb{C}_{\infty}$ denotes the Riemann sphere, and if $\Omega \subseteq \mathbb{C}_{\infty}$, then $\Omega^{*}$ denotes the reflection of $\Omega$ with 
respect to $\mathbb{T}$

$$
\Omega^{*}=\left\{\lambda \in \mathbb{C}_{\infty}: 1 / \bar{\lambda} \in \Omega\right\} .
$$

Thus $\mathbb{D}^{*}=\{z \in \mathbb{C}:|z|>1\} \cup\{\infty\}$. Let $f$ be analytic in $\mathbb{D}$ and let $G$ be meromorphic in $\mathbb{D}^{*}$. Suppose further that both $f$ and $G$ have nontangential limit values $f\left(e^{i t}\right)$ and $G\left(e^{i t}\right)$ almost everywhere on $\mathbb{T}$. The function $G$ is called a pseudocontinuation of $f$ across $\mathbb{T}$, if $f\left(e^{i t}\right)=G\left(e^{i t}\right)$ a.e. (see [6]).

If $\Omega \subseteq \mathbb{C}_{\infty}$ is a region, then the Nevanlinna class of $\Omega, N(\Omega)$, is the set of meromorphic functions on $\Omega$ that can be written as the quotient of two bounded functions. If $f \in N(\mathbb{D})$, then $f$ has nontangential limits a.e. on $\mathbb{T}$, which we shall denote by $f\left(e^{i t}\right)$. For a function $f \in D$ we shall write $z f$ and $(z f)^{\prime}$ for the functions $(z f)(\lambda)=\lambda f(\lambda)$ and $(z f)^{\prime}(\lambda)=f(\lambda)+\lambda f^{\prime}(\lambda)$, $\lambda \in \mathbb{D}$. Thus if $(z f)^{\prime} \in N(\mathbb{D})$, then $(z f)^{\prime}\left(e^{i t}\right)$ denotes the nontangential limit of $(z f)^{\prime}$, provided it exists.

In Theorem 2.2 we shall show that if $g \in D$ is orthogonal to some nonzero invariant subspace of $\left(M_{z}, D\right)$, then $(z g)^{\prime}$ is in the Nevanlinna class and there is a meromorphic function $G$ in $N\left(\mathbb{D}^{*}\right)$ which is a pseudocontinuation of $(z g)^{\prime}$ across $\mathbb{T}$. This gives a necessary condition on a function $g$ to be noncyclic for the adjoint of the Dirichlet shift. It is a partial analog of a theorem of Douglas, Shapiro, and Shields for $H^{2}$ [6].

If $f \in D$ and if $\mathscr{M}$ is an invariant subspace of $\left(M_{z}, D\right)$, then we use $\underline{Z}(f)$ and $\underline{Z}(\mathscr{M})$ to denote the lim inf zero sets of $f$ and $\mathscr{M}$ in the closed unit disc, i.e.,

$$
\underline{Z}(f)=\left\{z \in \overline{\mathbb{D}}: \liminf _{\lambda \rightarrow z, \lambda \in \mathbb{D}}|f(\lambda)|=0\right\}
$$

and

$$
\underline{Z}(\mathscr{M})=\bigcap_{f \in \mathscr{M}} \underline{Z}(f)
$$

Note that both sets are closed. Now let $\mathscr{M}$ be an invariant subspace and $g \perp$ $\mathscr{M}$. In $\S 3$ we show that the pseudocontinuation of $(z g)^{\prime}$ is analytic across any arc of $\mathbb{T} \backslash \underline{Z}(\mathscr{M})$. Furthermore, in Theorem 3.2 we shall see that for the extremal function $\varphi$ of the invariant subspace $\mathscr{M}$ one has $\underline{Z}(\varphi)=\underline{Z}(\mathscr{M})$, and $(z \varphi)^{\prime}$ is unbounded near any point in $\mathbb{T} \cap \underline{Z}(\mathscr{M})$. Thus $\underline{Z}(\mathscr{M})$ is the minimal set that has to be removed from the unit circle in order to get a meromorphic continuation. We then use these results to prove two corollaries. First we improve a result of Hedenmalm and Shields by showing that if $f \in D$ is an outer function such that $\underline{Z}(f)$ is countable, then $f$ must be cyclic in $D$, i.e., the polynomial multiples of $f$ are dense in $D$. Hedenmalm and Shields need to assume that $f$ is also in the disc algebra (see [10]). Secondly, in Corollary 3.4 we show that the spectrum of the compression of the Dirichlet shift to $\mathscr{M}^{\perp}$ equals $\underline{Z}(\mathscr{M})$. This is a direct analog of the well-known result for $H^{2}$.

In $\S 4$ we shall give an example of an invariant subspace $\mathscr{M}$ containing an outer function and such that $\underline{Z}(\mathscr{M})=\mathbb{T}$. This implies that the pseudocontinuations of $(z g)^{\prime}$ of functions $g \in \mathscr{M}^{\perp}$ may not be analytic near any point of the unit circle.

In $\S 5$ we prove a few results specifically for extremal functions. It turns out that both the multiplier- and the $H^{\infty}$-norm of the extremal function $\varphi$ are bounded by its Dirichlet norm. Furthermore, the radial limit of $|\varphi|$ exists at every point of the unit circle, and the resulting function on the closed unit disc 
is upper semicontinuous. It follows that the radial zero set of $\varphi, Z(\varphi)=\{z \in$ $\left.\overline{\mathbb{D}}: \lim _{r \rightarrow 1} f(r z)=0\right\}$, is a $G_{\delta}$ set.

\section{Pseudocontinuations}

In our proofs we shall make extensive use of computations that involve the local Dirichlet integral. Let $\zeta \in \mathbb{T}$ and $f \in H^{2}$. In [16, §2] it was shown that if

$$
\int_{\mathbf{T}}\left|\frac{f(w)-\alpha}{w-\zeta}\right|^{2} \frac{|d w|}{2 \pi}<\infty
$$

for some complex number $\alpha$, then the oricyclic limit (and therefore the nontangential limit) of $f$ at $\zeta$ exists and equals $\alpha$. Thus as in [16] we may define the local Dirichlet integral of $f$ at $\zeta$ by

$$
D_{\zeta}(f)=\int_{\mathbf{T}}\left|\frac{f(w)-f(\zeta)}{w-\zeta}\right|^{2} \frac{|d w|}{2 \pi} .
$$

It follows from a formula of Douglas for the Dirichlet integral [5] that

$$
D(f)=\int_{\mathbf{T}} D_{\zeta}(f) \frac{|d \zeta|}{2 \pi}
$$

Thus if $f \in D$ then $D_{\zeta}(f)$ is an $L^{1}$-function. We shall also use the mixed local Dirichlet integral. We define $D_{\zeta}(f, g)$ for $f, g \in H^{2}$ and $\zeta \in \mathbb{T}$ such that $D_{\zeta}(f), D_{\zeta}(g)<\infty$ by

$$
D_{\zeta}(f, g)=\int_{\mathbf{T}} \frac{(f(w)-f(\zeta))(\overline{g(w)-g(\zeta)})}{|w-\zeta|^{2}} \frac{|d w|}{2 \pi}
$$

Thus $D_{\zeta}(f, g) \in L^{1}$ whenever $f, g \in D$. For more information on the local Dirichlet integral we refer the reader to [16] and [17].

The following rather trivial lemma contains computational results which will be used repeatedly throughout the paper.

Lemma 2.1. Let $f, g \in D, \lambda \in \mathbb{D}$, and $\alpha, \beta \in \mathbb{C}$.

$$
\begin{aligned}
\left(\frac{1}{1-\bar{\lambda} z} f, g\right)_{D}= & \int_{\mathrm{T}} \frac{1}{1-\bar{\lambda} z}\left(f(z) \overline{g(z)}+D_{z}(f, g)\right) \frac{|d z|}{2 \pi} \\
& +\int_{\mathbf{T}} \frac{\bar{\lambda} z}{(1-\bar{\lambda} z)^{2}} f(z) \overline{g(z)} \frac{|d z|}{2 \pi},
\end{aligned}
$$

and

$$
\int_{\mathbb{T}} \frac{\bar{\lambda} z}{(1-\bar{\lambda} z)^{2}} f(z) \overline{g(z)} \frac{|d z|}{2 \pi}=\int_{\mathbf{T}} \frac{\bar{\lambda} z}{(1-\bar{\lambda} z)^{2}}(f(z)-\alpha) \overline{(g(z)-\beta)} \frac{|d z|}{2 \pi}+\alpha \overline{\bar{\lambda} g^{\prime}(\lambda)} .
$$


Proof. For $z \in \mathbb{T}$ with $D_{z}(g)<\infty$ we have

$$
\begin{aligned}
D_{z}\left(\frac{1}{1-\bar{\lambda} w} f, g\right)= & \int_{\mathbb{T}} \frac{\left((1-\bar{\lambda} z)^{-1} f(z)-(1-\bar{\lambda} w)^{-1} f(w)\right)(\overline{g(z)-g(w)})}{|z-w|^{2}} \frac{|d w|}{2 \pi} \\
= & \int_{\mathbb{T}} \frac{(f(z)-f(w))(\overline{g(z)-g(w)})}{(1-\bar{\lambda} z)|z-w|^{2}} \frac{|d w|}{2 \pi} \\
& +\int_{\mathbb{T}} f(w) \frac{((1-\bar{\lambda} w)-(1-\bar{\lambda} z))(\overline{g(z)-g(w)})}{(1-\bar{\lambda} z)(1-\bar{\lambda} w)|z-w|^{2}} \frac{|d w|}{2 \pi} \\
= & \frac{1}{1-\bar{\lambda} z} D_{z}(f, g)+\bar{\lambda} \int_{\mathbb{T}} \frac{f(w)}{1-\bar{\lambda} w} \frac{1}{1-\bar{\lambda} z} \frac{\overline{g(z)-g(w)}}{\bar{z}-\bar{w}} \frac{|d w|}{2 \pi} .
\end{aligned}
$$

Thus,

$$
\begin{aligned}
\left(\frac{1}{1-\bar{\lambda} z} f, g\right)_{D}= & \int_{\mathbb{T}} \frac{1}{1-\bar{\lambda} z} f(z) \overline{g(z)} \frac{|d z|}{2 \pi}+\int_{\mathbb{T}} D_{z}\left(\frac{1}{1-\bar{\lambda} w} f, g\right) \frac{|d z|}{2 \pi} \\
= & \int_{\mathbb{T}} \frac{1}{1-\bar{\lambda} z}\left(f(z) \overline{g(z)}+D_{z}(f, g)\right) \frac{|d z|}{2 \pi} \\
& +\bar{\lambda} \int_{\mathbb{T}} \frac{f(w)}{1-\bar{\lambda} w}\left(\int_{\mathbb{T}} \frac{1}{1-\bar{\lambda} z}\left(\frac{\overline{g(z)-g(w)}}{\bar{z}-\bar{w}}\right) \frac{|d z|}{2 \pi}\right) \frac{|d w|}{2 \pi} \\
= & \int_{\mathbb{T}} \frac{1}{1-\bar{\lambda} z}\left(f(z) \overline{g(z)}+D_{z}(f, g)\right) \frac{|d z|}{2 \pi} \\
& +\bar{\lambda} \int_{\mathbb{T}} \frac{f(w)}{1-\bar{\lambda} w} \frac{\overline{g(\lambda)-g(w)}}{\bar{\lambda}-\bar{w}} \frac{|d w|}{2 \pi} \\
= & \int_{\mathbb{T}} \frac{1}{1-\bar{\lambda} z}\left(f(z) \overline{g(z)}+D_{z}(f, g)\right) \frac{|d z|}{2 \pi} \\
& +\int_{\mathbb{T}} \frac{\bar{\lambda} w}{(1-\bar{\lambda} w)^{2}} f(w) \overline{g(w)} \frac{|d w|}{2 \pi} .
\end{aligned}
$$

An even more trivial computation yields the second identity of the lemma.

In the following we shall use $N^{+}\left(\mathbb{D}^{*}\right)$ to denote the Smirnov class of the exterior disc, i.e., those functions in $N\left(\mathbb{D}^{*}\right)$ that can be written as $f / g$, where $f, g \in H^{\infty}$ and $g$ is an outer function. For $p>0, H^{p} \subseteq N^{+}(\mathbb{D})$ is the classical Hardy space of analytic functions $f$ on $\mathbb{D}$ such that

$$
\|f\|_{p}^{P}=\sup _{r<1} \int_{\mathbb{T}}|f(r w)|^{p} \frac{|d w|}{2 \pi}<\infty .
$$

Theorem 2.2. Let $\mathscr{M} \in \operatorname{Lat}\left(M_{z}, D\right), \mathscr{M} \neq(0)$, and $g \in D, g \perp \mathscr{M}$. Then

(a) $(z g)^{\prime} \in N(\mathbb{D})$. In fact, if $f \in \mathscr{M}$ and $0<p<1$, then $f(z g)^{\prime} \in H^{p}$.

(b) $(z g)^{\prime}$ has a pseudocontinuation $G$ across $\mathbb{T} . G \mid \mathbb{D}^{*}$ is in the Nevanlinna class. If $\mathscr{M}$ contains an outer function, then $G \mid \mathbb{D}^{*} \in N^{+}\left(\mathbb{D}^{*}\right)$. The poles of $G \mid \mathbb{D}^{*}$ are contained in the set of points $\lambda \in \mathbb{D}^{*}$ such that $f(1 / \bar{\lambda})=0$ for all $f \in \mathscr{M}$.

Recall that if $\mathscr{H}$ is a Hilbert space and if $T$ is a bounded linear operator on $\mathscr{H}$, then a vector $x \in \mathscr{H}$ is called cyclic for $T$, if the set $\{p(T) x: p$ a polynomial $\}$ is dense in $\mathscr{H}$. Equivalently, $x$ is not cyclic for $T$ if and only if $x$ is orthogonal to some nonzero $T^{*}$-invariant subspace $\mathscr{M}$. Thus Theorem 2.2 
gives a necessary condition for $g$ to be noncyclic for $\left(M_{z}^{*}, D\right)$. In Propositions 2.3 and 2.4 we shall give two sufficient conditions for functions to be noncyclic for $\left(M_{z}^{*}, D\right)$.

We mentioned in the Introduction that this theorem is a partial analog of a result of [6], where it is proved that a function $g \in H^{2}$ is not cyclic for the backward unilateral shift, $\left(M_{z}^{*}, H^{2}\right)$, if and only if $g$ has a pseudocontinuation in $N\left(\mathbb{D}^{*}\right)$. Comparing this with our result one might wonder what the significance of the function $(z g)^{\prime}$ is. One indication that $(z g)^{\prime}$ is the right function to consider is given by the fact that

$$
(f, g)_{D}=\lim _{r \rightarrow 1^{-}} \frac{1}{2 \pi} \int_{0}^{2 \pi} f\left(r e^{i t}\right) \overline{(z g)^{\prime}\left(r e^{i t}\right)} d t \quad \forall f, g \in D .
$$

However, if one tried to use this formula and mimic the proof of [6] one runs into problems, because in general $(z g)^{\prime} \notin H^{1}$ (see the remark after the following proof).

Proof of Theorem 2.2. (a) Let $f \in \mathscr{M}$. Then $f g \in H^{1}$, thus it will suffice to show that the function defined by $f(\lambda) \lambda g^{\prime}(\lambda), \lambda \in \mathbb{D}$, is in $H^{P}$ for every $0<p<1$.

Because $g \perp z^{n} f$ for all $n \geq 0$, it follows from Lemma 2.1 that for each $\lambda \in \mathbb{D}$

$$
\begin{aligned}
0=\left(\frac{1}{1-\bar{\lambda} z} f, g\right)_{D}= & \int_{\mathrm{T}} \frac{1}{1-\bar{\lambda} z}\left(f(z) \overline{g(z)}+D_{z}(f, g)\right) \frac{|d z|}{2 \pi} \\
& +\int_{\mathbb{T}} \frac{\bar{\lambda} z}{(1-\bar{\lambda} z)^{2}} f(z) \overline{g(z)} \frac{|d z|}{2 \pi} .
\end{aligned}
$$

Thus we have for $\lambda \in \mathbb{D}$

$$
\begin{aligned}
\left|f(\lambda) \lambda g^{\prime}(\lambda)\right| \leq & \left|f(\lambda) \overline{\lambda g^{\prime}(\lambda)}-\int_{\mathrm{T}} \frac{\bar{\lambda} z}{(1-\bar{\lambda} z)^{2}} f(z) \overline{g(z)} \frac{|d z|}{2 \pi}\right| \\
& +\left|\int_{\mathrm{T}} \frac{1}{1-\bar{\lambda} z}\left(f(z) \overline{g(z)}+D_{z}(f, g)\right) \frac{|d z|}{2 \pi}\right| .
\end{aligned}
$$

The function $(f \bar{g})(z)+D_{z}(f, g)$ is in $L^{1}(\mathbb{T})$, hence the complex conjugate of $\int_{\mathrm{T}}(1-\bar{\lambda} z)^{-1}\left(f(z) \overline{g(z)}+D_{z}(f, g)\right)|d z| / 2 \pi$ is in $H^{p}, 0<p<1$, see [7, $\mathbf{p}$. 39]. This means that the proof of (a) will be completed once we show that

$$
\left|f(\lambda) \overline{\lambda g^{\prime}(\lambda)}-\int_{\mathbf{T}} \frac{\bar{\lambda} z}{(1-\bar{\lambda} z)^{2}} f(z) \overline{g(z)} \frac{|d z|}{2 \pi}\right| \leq h(w), \quad w=\frac{\lambda}{|\lambda|},
$$

for some $L^{1}(\mathbb{T})$-function $h$.

Now use the second identity of Lemma 2.1 with $\alpha=f(\lambda)$ and $\beta=g(\lambda)$ to 
obtain

$$
\begin{aligned}
\left|f(\lambda) \overline{\lambda g^{\prime}(\lambda)}-\int_{\mathbb{T}} \frac{\bar{\lambda} z}{(1-\bar{\lambda} z)^{2}} f(z) \overline{g(z)} \frac{|d z|}{2 \pi}\right| \\
\quad=\mid \int_{\mathbb{T}} \frac{\bar{\lambda} z}{(1-\bar{\lambda} z)^{2}}\left(f(z)-f(\lambda) \overline{(g(z)-g(\lambda))} \frac{|d z|}{2 \pi} \mid\right. \\
\quad \leq\left(\int_{\mathbb{T}} \frac{|f(z)-f(\lambda)|^{2}}{|z-\lambda|^{2}} \frac{|d z|}{2 \pi}\right)^{1 / 2}\left(\int_{\mathbb{T}} \frac{|g(z)-g(\lambda)|^{2}}{|z-\lambda|^{2}} \frac{|d z|}{2 \pi}\right)^{1 / 2} \\
\leq 4 D_{w}(f)^{1 / 2} D_{w}(g)^{1 / 2}, \quad w=\frac{\lambda}{|\lambda|},
\end{aligned}
$$

by Lemma 3.3(a) of [16]. This concludes the proof of (a), because the assumptions that $f, g \in D$ imply that $D_{w}(f)^{1 / 2} D_{w}(g)^{1 / 2} \in L^{1}(\mathbb{T})$.

(b) Let $f \in \mathscr{M}, f \neq 0$. For $\lambda \in \mathbb{D}$ define

$$
F(\lambda)=\int_{\mathbb{T}} \frac{\lambda \bar{z}}{1-\lambda \bar{z}}\left(f(z) \overline{g(z)}+D_{z}(f, g)\right) \frac{|d z|}{2 \pi} .
$$

Then $F$ is the Cauchy transform of a finite measure, hence $F \in H^{p}$ for all $0<p<1$ [7, p. 39], and it has a nontangential limit function $F(w)$ which is defined and finite at almost every $w \in \mathbb{T}$. We shall show that

$$
F(w)=f(w) \overline{(z g)^{\prime}(w)} \text { a.e. } w \in \mathbb{T} \text {. }
$$

Then the function $G(\lambda)=\overline{F(1 / \bar{\lambda}) / f(1 / \bar{\lambda})}$ will be the meromorphic pseudocontinuation of $(z g)^{\prime}$ to $\mathbb{D}^{*}$. Notice that by the uniqueness of the pseudocontinuation (see [6, p. 39]) the function $G$ is independent of $f$. Furthermore, we see that $G \in N\left(\mathbb{D}^{*}\right)$, and even $G \in N^{+}\left(\mathbb{D}^{*}\right)$, if $f$ can be chosen to be an outer function. We also observe that $G$ can only have a pole at $\lambda \in \mathbb{D}^{*}$ if $f(1 / \bar{\lambda})=0$ for all $f \in \mathscr{M}$.

Fix $w \in \mathbb{T}$ such that $D_{w}(f), D_{w}(g)<\infty$. Then $f(w)$ and $g(w)$ exist as finite nontangential limits of $f(\lambda)$ and $g(\lambda), \lambda \in \mathbb{D}$ (see $[16, \S 2]$ ). Note that the Poisson kernel $P_{\lambda}$ equals $P_{\lambda}(z)=\lambda \bar{z} /(1-\lambda \bar{z})+1 /(1-\bar{\lambda} z),|z|=1$. Now we use both statements of Lemma 2.1 (choose $\alpha=f(w)$ and $\beta=g(w)$ ) together with the fact that $\left((1-\bar{\lambda} z)^{-1} f, g\right)_{D}=0$, and we obtain for $\lambda \in \mathbb{D}$

$$
\begin{aligned}
\mid F(\lambda) & -f(w) \overline{(z g)^{\prime}(\lambda)} \mid \\
= & \left|\int_{\mathbb{T}} \frac{\lambda \bar{z}}{1-\lambda \bar{z}}\left(f(z) \overline{g(z)}+D_{z}(f, g)\right) \frac{|d z|}{2 \pi}-f(w) \overline{g(\lambda)}-f(w) \overline{\lambda g^{\prime}(\lambda)}\right| \\
= & \mid \int_{\mathbf{T}} P_{\lambda}(z)\left(f(z) \overline{g(z)}+D_{z}(f, g)\right) \frac{|d z|}{2 \pi}-f(w) \overline{g(\lambda)} \\
& \quad+\int_{\mathbb{T}} \frac{\bar{\lambda} z}{(1-\bar{\lambda} z)^{2}}(f(z)-f(w)) \overline{(g(z)-g(w))} \frac{|d z|}{2 \pi} \mid
\end{aligned}
$$

We note that by Fatou's theorem the sum of the first two summands of this last expression converges to $D_{w}(f, g)$ for a.e. $w \in \mathbb{T}$ as $\lambda \rightarrow w$ nontangentially. The integral on the right of this expression equals

$$
\int_{\mathbf{T}} \frac{\bar{\lambda} z}{(1-\bar{\lambda} z)^{2}}|z-w|^{2} \frac{(f(z)-f(w)) \overline{(g(z)-g(w))}}{|z-w|^{2}} \frac{|d z|}{2 \pi}
$$


and, because $\bar{\lambda} z /(1-\bar{\lambda} z)^{-2}|z-w|^{2} \rightarrow-1$ as $\lambda \rightarrow w$, it easily follows from the dominated convergence theorem that it converges to $-D_{w}(f, g)$ as $\lambda \rightarrow w$ nontangentially. This concludes the proof of equation (2.2) and the proof of Theorem 2.2.

We remark that part (a) of Theorem 2.2 cannot be improved to state that $(z g)^{\prime} \in H^{1}$. In fact, let us show that, if $g \in \mathscr{M}^{\perp},(z g)^{\prime} \in H^{1}$, and if $\mathscr{M}^{\prime}$ contains an outer function, then $g=0$.

By Theorem 3.1 of [17] we may assume that $\mathscr{M}$ contains a bounded outer function $f$. Under these assumptions we can use formula (2.1) to see that

$$
0=\left(z^{n} f, g\right)_{D}=\int_{\mathbb{T}} z^{n} f(z) \overline{(z g)^{\prime}(z)} \frac{|d z|}{2 \pi} \quad \forall n \geq 0 .
$$

Thus, the $L^{1}(\mathbb{T})$-function $\overline{(z g)^{\prime}}$ is in the preannihilator of the weak ${ }^{*}$ closed ideal generated by $f$ in $H^{\infty}$. Since $f$ is outer, this ideal coincides with $H^{\infty}$. Hence

$$
0=\int_{\mathbb{T}} z^{n} \overline{(z g)^{\prime}(z)} \frac{|d z|}{2 \pi} \quad \forall n \geq 0,
$$

this implies that $g=0$.

Inspection of the proof of Theorem 2.2 (see equation (2.2)) shows that if $g \in D$ is not a cyclic vector for $\left(M_{z}^{*}, D\right)$, then $(z g)^{\prime} \in N(\mathbb{D})$ and one even has that $\overline{(z g)^{\prime}(w)}=F(w) / f(w)$ a.e. $w \in \mathbb{T}$, where $F$ is the Cauchy transform of an $L^{1}$-function and $f \in D \cap H^{\infty}$. In the converse direction one easily obtains the following sufficient conciition for a vector to be noncyclic for $\left(M_{z}^{*}, D\right)$. Recall that $H^{1} \subseteq L_{a}^{2}(\mathbb{D})$ [18, p. 325]; also see [1, p. 275].

Proposition 2.3. Let $g \in D$ such that $(z g)^{\prime} \in H^{1}$. Then $g$ is not cyclic for $\left(M_{z}^{*}, D\right)$ if and only if $\overline{(z g)^{\prime}(w)}=F(w) / f(w)$ a.e. $w \in \mathbb{T}$ for some $F \in N^{+}(\mathbb{D})$ and $f \in D$.

Proof. As mentioned before the only if direction follows from Theorem 2.2. By Theorem 1 of [15] every function in $D$ can be written as the quotient of two bounded functions in $D$. Thus for the converse we may assume that $f \neq 0$, $f \in D \cap H^{\infty}$. Then the assumption $(z g)^{\prime} \in H^{1}$ implies that $F \in H^{1}$ as well. By use of formula (2.1) we see that for $n \geq 0$

$$
\left(z^{n} f, g\right)_{D}=\int_{\mathbb{T}} w^{n} f(w) \overline{(z g)^{\prime}(w)} \frac{|d w|}{2 \pi}=\int_{\mathbb{T}} w^{n} F(w) \frac{|d w|}{2 \pi}=0 .
$$

Thus $g$ is orthogonal to a nonzero invariant subspace of the Dirichlet shift, and hence is not cyclic for $\left(M_{z}^{*}, D\right)$.

Proposition 2.3 covers some obvious classes of noncylic vectors of $\left(M_{z}^{*}, D\right)$ such as finite linear combinations of kernel functions (their derivatives are rational functions with poles off $\overline{\mathbb{D}}$ ). However, the following examples are not covered. is

Let $\mu$ be a positive finite Borel measure on $\mathbb{T}$, then the energy integral of $\mu$

$$
I(\mu)=\int_{\mathbb{T}} \int_{\mathbb{T}} \log \frac{1}{|w-z|} d \mu(w) d \mu(z) .
$$

One checks that the Cauchy transform $C_{\mu}(\lambda)=\int_{\mathbb{T}}(1-\lambda \bar{z})^{-1} d \mu(z), \lambda \in \mathbb{D}$, is in the Bergman space $L_{a}^{2}(\mathbb{D})$ if and only if $I(\mu)<\infty$ (see, e.g., [1, p. 294]). 
Recall that a set $E \subseteq \mathbb{T}$ has positive logarithmic capacity if there exists a positive finite Borel measure $\mu$ with support in $E$ and $I(\mu)<\infty$. Beurling showed that if $f \in D$, then the radial limit of $f$ exists everywhere except possibly on a set of logarithmic capacity 0 . Thus, if $I(\mu)<\infty$, then $f\left(e^{i t}\right)$ exists except on a set of $\mu$ measure 0 , and if $f \in D \cap H^{\infty}$ and $g \in D$ such that $(z g)^{\prime}=C_{\mu}$, then it follows from Fubini's theorem and the dominated convergence theorem that

$$
(f, g)_{D}=\lim _{r \rightarrow 1} \int_{\mathbb{T}} f(r w) \overline{C_{\mu}(r w)} \frac{|d w|}{2 \pi}=\int_{\mathbb{T}} f(z) d \mu(z) .
$$

Proposition 2.4. Let $\mu$ be a finite Borel measure on $\mathbb{T}$ with finite energy integral and which is singular with respect to Lebesgue measure on $\mathbb{T}$. Let $g \in D$ such that $(z g)^{\prime}=C_{\mu}$. Then $g$ is not cyclic for $\left(M_{z}^{*}, D\right)$ if and only if there exists a nonzero function $f \in D$ such that $\mu$ is concentrated in the radial zero set $Z(f)$.

Proof. If $f \in D \cap H^{\infty}$ and $n \geq 0$, then by the above

$$
\left(z^{n} f, g\right)_{D}=\int_{\mathbb{T}} z^{n} f(z) d \mu(z)
$$

Hence since $\mu$ is singular it follows from the F. and M. Riesz theorem that $g$ is not cyclic for $\left(M_{z}^{*}, D\right)$ if and only if there exists an $f \in D \cap H^{\infty}, f \neq 0$, such that the measure $f d \mu=0$. This is just another way of saying that $\mu$ is concentrated in $Z(f)$. Thus, to finish the proof it will suffice to note that if $f \in D$, then $f=\varphi / \psi$ for $\varphi, \psi \in D \cap H^{\infty}$ [15, Theorem 1], and the radial zero set of $\varphi, Z(\varphi)$, is larger than $Z(f)$.

\section{Analytic continuations}

Recall that for $f \in D$ we denote by $\underline{Z}(f)$ the lim inf zero set of $|f|$ in the closed unit disc. In $\S 2$ we saw that if $g \perp \mathscr{M}$ for some nonzero invariant subspace, then $(z g)^{\prime}$ has a pseudocontinuation to $\mathbb{D}^{*}$. Furthermore, the pseudocontinuation is analytic in $\mathbb{D}^{*}$ except for possible poles at the points $\lambda \in \mathbb{D}^{*}$ such that $1 / \bar{\lambda} \in \underline{Z}(f)$ for all $f \in \mathscr{M}$, i.e., $\lambda \in \mathbb{D}^{*} \cap \underline{Z}(\mathscr{M})^{*}$, where as before $\underline{Z}(\mathscr{M})^{*}$ denotes the reflected set $\underline{Z}(\mathscr{M})^{*}=\left\{\lambda \in \mathbb{C}_{\infty}: 1 / \bar{\lambda} \in \underline{Z}(\mathscr{M})\right\}$. We shall see now that $(z g)^{\prime}$ also extends to be analytic across every open arc of $\mathbb{T} \backslash \underline{Z}(\mathscr{M})$.

If $\varphi \in \mathscr{M} \ominus z \mathscr{M}, \varphi \neq 0$, then as we mentioned in the Introduction $\mathscr{M}$ is generated by the extremal function $\varphi$. Clearly $\underline{Z}(\mathscr{M}) \subseteq \underline{Z}(\varphi)$, and we shall see that in fact $\underline{Z}(\mathscr{M})=\underline{Z}(\varphi)$. Furthermore, if $\mathscr{M}$ contains a function that does not vanish at the origin, then it is easy to see that there is a constant $c \in \mathbb{C}$ such that the function $c-\varphi(z)$ is in $\mathscr{M}^{\perp}$. Thus, $\varphi$ plays a special role in these considerations, as it carries the analytical properties of both the functions in $\mathscr{M}$ and in $\mathscr{M}^{\perp}$. In particular, $(z \varphi)^{\prime}$ extends to be analytic across every open arc of $\mathbb{T} \backslash \underline{Z}(\mathscr{M})$, and we shall see that it is unbounded near $\mathbb{T} \cap \underline{Z}(\mathscr{M})$. Hence $T \backslash \underline{Z}(\mathscr{M})$ is the maximal set such that whenever $g \in \mathscr{M}^{\perp}$, then $\left(z g^{\prime}\right)$ extends analytically across it.

Lemma 3.1. Let $\mathscr{M} \in \operatorname{Lat}\left(M_{z}, D\right), \mathscr{M} \neq(0)$.

(a) If $g \in \mathscr{M}^{\perp}$, then $(z g)^{\prime}$ extends to be analytic in $\mathbb{C}_{\infty} \backslash \underline{Z}(\mathscr{M})^{*}$.

(b) If $\varphi$ is an extremal function for $\mathscr{M}$, i.e., $\varphi \in \mathscr{M} \ominus z \mathscr{M}, \varphi \neq 0$, and if $\zeta \in \mathbb{T} \cap \underline{Z}(\varphi)$, then there is a sequence $\left\{\lambda_{n}\right\}_{n \in \mathbb{N}} \subseteq \mathbb{D},\left\{\lambda_{n}\right\} \rightarrow \zeta$, such that $\left|(z \varphi)^{\prime}\left(\lambda_{n}\right)\right| \rightarrow \infty$. 
Proof. (a) Let $g \in \mathscr{M}^{\perp}$ and $\zeta \in \mathbb{T} \backslash \underline{Z}(\mathscr{M}) \quad\left(=\mathbb{T} \backslash \underline{Z}(\mathscr{M})^{*}\right)$. Using the pseudocontinuation of Theorem 2.2(b) we only have to show that $(z g)^{\prime}$ extends to be analytic in a neighborhood of $\zeta$.

$\zeta \in \mathbb{T} \backslash \underline{Z}(\mathscr{M})$ implies that there exists $f \in \mathscr{M}$ and an open set $\mathscr{U}, \zeta \in \mathscr{U}$, such that $|f(z)| \geq 1$ for all $z \in \mathscr{U} \cap \mathbb{D}$. We shall first show that there is a function $\tilde{f} \in \mathscr{M}$ such that $\tilde{f}$ extends analytically across $I=\mathscr{U} \cap \mathbb{T}$. Let $f=B h$ be the inner-outer factorization of $f$. Then the Blaschke factor of $B$ has no zeros in $\mathscr{U}$, and the singular measure associated with the singular inner factor of $B$ does not put any mass on $I$. This is well known. A simple argument for the singular inner factor goes as follows: If $B=B_{1} S_{\sigma}$, where $\sigma$ is a singular measure with $\operatorname{supp} \sigma \subseteq I$, then it would follow that $\left|1 / S_{\sigma}\right|$ is less than or equal to $\left|B_{1} h\right|$ in $\mathscr{U}$ and it is uniformly bounded in $\mathscr{D} \mid \mathscr{U}$ (see [8, Theorem II.6.2]), hence $1 / S_{\sigma} \in H^{2}$ which is impossible for a nonzero $\sigma$. Hence $B$ does not have a nontrivial singular inner divisor $S_{\sigma}$ with supp $\sigma \subseteq I$, or, equivalently, the singular inner factor of $B$ does not put any mass on $I$.

Thus by Theorems II.6.1 and II.6.2 of [8] the inner factor $B$ of $f$ continues analytically across $I$, and the outer factor $h$ still satisfies $|h(z)| \geq 1$ for all $z \in \mathscr{U} \cap \mathbb{D}$. Now define a new outer function by $\left|h_{0}\left(e^{i t}\right)\right|=\min \left\{1,\left|h\left(e^{i t}\right)\right|\right\}$. Then $\left|h_{0}\right|=1$ a.e. on $I$. Hence the function

$$
h_{0}(z)=\exp \left\{\frac{1}{2 \pi} \int \frac{e^{i t}+z}{e^{i t}-z} \log \left|h_{0}\left(e^{i t}\right)\right| d t\right\}
$$

extends to be continuous with modulus one on $I$. Thus, by the Schwarz reflection principle $h_{0}$ extends to be analytic across $I$, and so does $\tilde{f}=B h_{0}$.

Furthermore, it was shown in [17, Lemma 2.6] that the cutoff function $\tilde{f}$ is in the Dirichlet space, and that in fact $\tilde{f} \in \mathscr{M}$.

Thus assume that $\mathscr{M}$ contains a function $f$ which extends to be analytic and nonzero in a neighborhood $\mathscr{U}$ of $\zeta$. By possibly decreasing the size of $\mathscr{U}$ we may assume that $\mathscr{U}=\mathscr{U}^{*}$. Now consider the function $F: \mathscr{U} \times \mathbb{D} \rightarrow \mathbb{C}$,

$$
F(\lambda, z)=\frac{1-f(z) / f(1 / \bar{\lambda})}{1-\bar{\lambda} z} .
$$

It is easy to check that for each fixed $\lambda \in \mathscr{U}$ the function $F(\lambda, \cdot)$ is in the Dirichlet space. Furthermore, as a function of $\lambda F$ is antiholomorphic; hence we can define an analytic function in $\mathscr{U}$ by setting $G(\lambda)=(g, F(\lambda, \cdot))_{D}$. Since $f \in \mathscr{M}$ and $g \in \mathscr{M}^{\perp}$ we have for each $\lambda \in \mathbb{D}$

$$
g \perp(1-\bar{\lambda} z)^{-1} f / f(1 / \bar{\lambda}),
$$

so that $G(\lambda)=(g, F(\lambda, \cdot))_{D}=(g, 1 /(1-\bar{\lambda} z))_{D}=(z g)^{\prime}(\lambda)$.

(b) Now consider a function $\varphi \in \mathscr{M} \ominus z \mathscr{M}, \varphi \neq 0$, and let $\zeta \in \mathbb{T} \cap \underline{Z}(\varphi)$. For any $\lambda \in \mathbb{D}$ we have $\|\varphi\|_{D}^{2}=(1 /(1-\bar{\lambda} z) \varphi, \varphi)_{D}$. Thus, by Lemma 2.1 we see that

$$
\|\varphi\|_{D}^{2}=\int_{\mathrm{T}} \frac{1}{1-\bar{\lambda} z}\left(|\varphi(z)|^{2}+D_{z}(\varphi)\right) \frac{|d z|}{2 \pi}+\int_{\mathrm{T}} \frac{\bar{\lambda} z}{(1-\bar{\lambda} z)^{2}}|\varphi(z)|^{2} \frac{|d z|}{2 \pi} .
$$

Next we take twice the real part of the previous equation and subtract $\|\varphi\|_{D}^{2}$ to obtain

$$
\|\varphi\|_{D}^{2}=\int_{\mathbb{T}} P_{\lambda}(z)\left(|\varphi(z)|^{2}+D_{z}(\varphi)\right) \frac{|d z|}{2 \pi}+2 \operatorname{Re} \int_{\mathbf{T}} \frac{\bar{\lambda} z}{(1-\bar{\lambda} z)^{2}}|\varphi(z)|^{\mid} \frac{|d z|}{2 \pi} .
$$


For a.e. $w \in \mathbb{T} \varphi(w)$ exists as a nontangential limit. For such $w$ we apply Lemma 2.1 with $\alpha=\beta=\varphi(w)$, and we see

$$
\begin{aligned}
\|\varphi\|_{D}^{2}= & \int_{\mathbb{T}} P_{\lambda}(z)\left(|\varphi(z)|^{2}+D_{z}(\varphi)\right) \frac{|d z|}{2 \pi} \\
& +2 \operatorname{Re} \int_{\mathbb{T}} \frac{\bar{\lambda} z}{(1-\bar{\lambda} z)^{2}}|\varphi(z)-\varphi(w)|^{2} \frac{|d z|}{2 \pi}+2 \operatorname{Re} \varphi(w) \overline{\lambda \varphi^{\prime}(\lambda)} .
\end{aligned}
$$

From the remark before Lemma 3.1 and Theorem 2.2 we know that $\varphi^{\prime} \in N$, thus $\varphi^{\prime}(w)$ exists for a.e. $w \in \mathbb{T}$ as the nontangential limit of $\varphi^{\prime}(\lambda), \lambda \in \mathbb{D}$. Furthermore, as $\lambda$ approaches $w$ nontangentially the second summand on the right of the previous equation converges by the dominated convergence theorem to $-2 D_{w}(\varphi)$, whenever $D_{w}(\varphi)$ is finite (as in the proof of Theorem 2.2). Hence by Fatou's theorem we obtain for a.e. $w \in \mathbb{T}$

$$
\|\varphi\|_{D}^{2}=-D_{w}(\varphi)-|\varphi(w)|^{2}+2 \operatorname{Re} \varphi(w) \overline{(z \varphi)^{\prime}(w)} .
$$

The Dirichlet space is contained in the space VMOA (see [21]), and for functions in VMOA the cluster set equals the essential range of the radial limit function at every point $w \in \mathbb{T}$ (see [20]). In particular, $\zeta \in \underline{Z}(\varphi)$ implies that there is a sequence $\left\{w_{n}\right\}_{n \in \mathbb{N}} \subseteq \mathbb{T}$ such that $\left\{w_{n}\right\} \rightarrow \zeta,\left\{\varphi\left(w_{n}\right)\right\} \rightarrow 0$, and every $w_{n}$ satisfies (3.2). But this can only be the case if $\left|(z \varphi)^{\prime}\left(w_{n}\right)\right| \rightarrow \infty$; hence there must also be a sequence $\left\{\lambda_{n}\right\} \subseteq \mathbb{D},\left\{\lambda_{n}\right\} \rightarrow \zeta$, and such that $\left|(z \varphi)^{\prime}\left(\lambda_{n}\right)\right| \rightarrow \infty$.

Theorem 3.2. Let $\mathscr{M} \in \operatorname{Lat}\left(M_{z}, D\right), \varphi \in \mathscr{M} \ominus z \mathscr{M}, \varphi \neq 0$.

Then $\underline{Z}(\varphi)=\underline{Z}(\mathscr{M})$. Futhermore, $\mathbb{C}_{\infty} \backslash \underline{Z}(\mathscr{M})^{*}$ is the largest set with the property that, whenever $g \in \mathscr{M}^{\perp}$, then $(z g)^{\prime}$ extends to be analytic in $\mathbb{C}_{\infty} \backslash \underline{Z}(\mathscr{M})^{*}$.

Proof. Clearly $\underline{Z}(\mathscr{M}) \subseteq \underline{Z}(\varphi)$ and $\underline{Z}(\mathscr{M}) \cap \mathbb{D}=\underline{Z}(\varphi) \cap \mathbb{D}$, because $\mathscr{M}$ is generated by $\varphi$. But if $\zeta \in \underline{Z}(\varphi) \cap \mathbb{T}$, then by part (b) of Lemma $3.1(z \varphi)^{\prime}$ cannot extend to be analytic in a neighborhood of $\zeta$. Thus by Lemma 3.1(a) and the remark made before Lemma $3.1 \zeta \notin \mathbb{C} \backslash \underline{Z}(\mathscr{M})^{*}$, hence $\zeta \in \underline{Z}(\mathscr{M})$.

According to the remark before Lemma 3.1 and Lemma 3.1(b), to conclude the proof of Theorem 3.2 it will be enough to establish that for each $\lambda \in \mathbb{D}^{*} \cap$ $\underline{Z}(\mathscr{M})^{*}$ there is a function $g \in \mathscr{M}^{\perp}$ such that $(z g)^{\prime}$ has a pole at $\lambda$. But this is trivial since $\lambda \in \mathbb{D}^{*} \cap \underline{Z}(\mathscr{M})^{*}$ implies that $\mu=1 / \bar{\lambda} \in \underline{Z}(\mathscr{M}) \cap \mathbb{D}$, so that the reproducing kernel $k_{\mu}(z)=(1 / \bar{\mu} z) \log (1 /(1-\bar{\mu} z)) \in \mathscr{M}^{\perp}$ and $\left(z k_{\mu}\right)^{\prime}(z)=$ $1 /(1-\bar{\mu} z)$ has a pole at $\lambda$.

What are the sets that one can obtain as $\underline{Z}(\mathscr{M})$ of some invariant subspace $\mathscr{M}$ ? The function $f(z)=(1-z) \exp \{-(1+z) /(1-z)\}$ is not cyclic in $D$, and if $[f]$ denotes the smallest invariant subspace containg $f$, then $\underline{Z}([f])=\{1\}$. However, we shall see now that if $\mathscr{M}$ contains an outer function, then $\underline{Z}(\mathscr{M})$ cannot be arbitrarily small.

If $\mathscr{X}$ is a space of analytic functions in $\mathbb{D}$ and $\mathscr{Y}$ is a space of analytic functions in $\mathbb{D}^{*}$, then we say that a closed set $E \subseteq \mathbb{T}$ is an $(\mathscr{X}, \mathscr{Y})$ exceptional set, if there are nonconstant functions $f$ that are holomorphic in $\mathbb{C}_{\infty} \backslash E$ and such that $f \mid \mathbb{D} \in \mathscr{X}$ and $f \mid \mathbb{D}^{*} \in \mathscr{Y}$. A theorem of Carleson implies that the $\left(L_{a}^{2}(\mathbb{D}), L_{a}^{2}\left(\mathbb{D}^{*}\right)\right)$ exceptional sets are exactly the closed subsets of $\mathbb{T}$ of positive logarithmic capacity (see $[4$, p. 73$]$ ). 
Corollary 3.3. Let $\mathscr{M} \in \operatorname{Lat}\left(M_{z}, D\right), \mathscr{M} \neq D$. If $\mathscr{M}$ contains an outer function, then $\underline{Z}(\mathscr{M})$ is an $\left(L_{a}^{2}(\mathbb{D}) \cap N(\mathbb{D}), N^{+}\left(\mathbb{D}^{*}\right)\right)$ exceptional set. In fact, if $\mathscr{U} \subseteq \mathbb{T}$ is open and $\underline{Z}(\mathscr{M}) \cap \mathscr{U}$ is nonempty, then $\underline{Z}(\mathscr{M}) \cap \mathscr{U}$ contains an $\left(L_{a}^{2}(\mathbb{D}) \cap\right.$ $N(\mathbb{D}), N^{+}\left(\mathbb{D}^{*}\right)$ ) exceptional set.

This theorem improves a result of Hedenmalm and Shields [10, Theorem 2], who show that if $\mathscr{M} \neq D$ and if $\mathscr{M} \cap A$ contains an outer function, then $\underline{Z}(\mathscr{M} \cap A)$ is an $\left(L_{a}^{2}(\mathbb{D}), N^{+}\left(\mathbb{D}^{*}\right)\right)$ exceptional set. Here we use $A$ to denote the disc algebra of functions in $H^{\infty}$ which extend to be continuous on $\overline{\mathbb{D}}$. Also compare Proposition 2 of [10]. Actually Hedenmalm and Shields have the roles of $\mathbb{D}$ and $\mathbb{D}^{*}$ reversed, but the results are equivalent as they use $L_{a}^{2}\left(\mathbb{D}^{*}\right)$ to denote the Bergman space with respect to area measure on the Riemann sphere (a finite measure!).

Hedenmalm and Shields show that countable closed sets are not $\left(L_{a}^{2}(\mathbb{D})\right.$, $\left.N^{+}\left(\mathbb{D}^{*}\right)\right)$ exceptional sets. Thus Corollary 3.3 implies that any outer function $f \in D$ such that $\underline{Z}(f)$ is countable must be cyclic in $D$.

Proof of Corollary 3.3. Since $\mathscr{M}$ contains an outer function we have $\underline{Z}(\mathscr{M})^{*}=$ $\underline{Z}(\mathscr{M}) \subseteq \mathbb{T}$. Let $\varphi \in \mathscr{M} \ominus z \mathscr{M}, \varphi \neq 0$. By Theorems 2.2 and $3.2(z \varphi)^{\prime}$ has an analytic extension $G$ to $\mathbb{C}_{\infty} \backslash \underline{Z}(\mathscr{M})$ such that $G \mid \mathbb{D}^{*}$ is in the Smirnov class and $G \mid \mathbb{D}=(z \varphi)^{\prime} \in L_{a}^{2}(\mathbb{D}) \cap N(\mathbb{D})$. The assumption $\mathscr{M} \neq D$ implies that $(z \varphi)^{\prime}$ is not constant, hence $\underline{Z}(\mathscr{M})$ is an $\left(L_{a}^{2}(\mathbb{D}) \cap N(\mathbb{D}), N^{+}\left(\mathbb{D}^{*}\right)\right)$ exceptional set.

Now let $\mathscr{U} \subseteq \mathbb{T}$ be open and suppose that $\underline{Z}(\mathscr{M}) \cap \mathscr{U}$ is nonempty. Nonempty closed intervals are certainly $\left(L_{a}^{2}(\mathbb{D}) \cap N(\mathbb{D}), N^{+}\left(\mathbb{D}^{*}\right)\right)$ exceptional sets, hence we may assume that $\underline{Z}(\mathscr{M}) \cap \mathscr{U}$ is totally disconnected. It follows that there is a nonempty compact set $E \subseteq \underline{Z}(\mathscr{M}) \cap \mathscr{U}$ such that the set $F=\underline{Z}(\mathscr{M}) \backslash E$ is also compact. We shall show that $E$ is an $\left(L_{a}^{2}(\mathbb{D}) \cap N(\mathbb{D}), N^{+}\left(\mathbb{D}^{*}\right)\right)$ exceptional set.

By use of the Cauchy integral we may write $G$ as $G=G_{1}+G_{2}$, where $G_{1}$ is holomorphic on the complement of $E$, and $G_{2}$ is holomorphic on the complement of $F$. That $G_{i} \mid \mathbb{D} \in L_{a}^{2}(\mathbb{D}) \cap N(\mathbb{D})$ and $G_{i} \mid \mathbb{D}^{*} \in N^{+}\left(\mathbb{D}^{*}\right), i=1,2$, follows exactly as in the proof of Proposition 2 of [10]. According to Lemma 3.1 $(z \varphi)^{\prime}=G \mid \mathbb{D}$ does not extend to be analytic in a neighborhood of any point of $\underline{Z}(\mathscr{M})$. Thus $G_{1}$ does not extend to be analytic in a neighborhood of any point of $E$. Hence $G_{1}$ cannot be constant, i.e. $E$ is an $\left(L_{a}^{2}(\mathbb{D}) \cap N(\mathbb{D}), N^{+}\left(\mathbb{D}^{*}\right)\right)$ exceptional set.

Let $T$ denote the compression of the Dirichlet shift to $\mathscr{M}^{\perp}$, i.e., if $P$ is the projection of $D$ onto $\mathscr{M}^{\perp}$, then $T=P M_{z} \mid \mathscr{M}^{\perp}$. The following result is an analog of a well-known result for $H^{2}$ [11].

Corollary 3.4. Let $\mathscr{M} \in \operatorname{Lat}\left(M_{z}, D\right), \varphi \in \mathscr{M} \ominus z \mathscr{M}, \varphi \neq 0$. Then

$$
\sigma(T)=\underline{Z}(\mathscr{M})=\underline{Z}(\varphi) .
$$

Proof. From Theorem 3.2 we know that $\underline{Z}(\mathscr{M})=\underline{Z}(\varphi)$. Since $\mathscr{M}=[\varphi]$ it is easy to see that $\sigma(T) \cap \mathbb{D}=\underline{Z}(\mathscr{M}) \cap \mathbb{D}$ (see [12, Theorem 4.5(a)]). Furthermore, it is clear that $\sigma(T), \underline{Z}(\mathscr{M}) \subseteq \overline{\mathbb{D}}$. Thus, we have to show that $\sigma(T) \cap \mathbb{T}=$ $\underline{Z}(\mathscr{M}) \cap \mathbb{T}$. Note that $T^{*}=M_{z}^{*} \mid \mathscr{M}^{\perp}$.

First suppose that $\zeta \in \mathbb{T} \backslash \sigma(T)$. Then there is a neighborhood $\mathscr{U}$ of $\zeta$ such that $\left(M_{z}-\lambda\right)^{*} \mid \mathscr{M}^{\perp}$ is invertible for every $\lambda \in \mathscr{U}$. We may assume that $\mathscr{U}=\mathscr{U}^{*}$. Thus for every $g \in \mathscr{M}^{\perp}, G(\lambda)=\left(\left(1-\bar{\lambda} M_{z}\right)^{*-1} g, P 1\right)_{D}$ defines an 
analytic function in $\mathscr{U}$. For $\lambda \in \mathbb{D}\left(1-\bar{\lambda} M_{z}\right)^{*}$ is invertible on $D$, hence

$$
G(\lambda)=\left(\left(1-\bar{\lambda} M_{z}\right)^{*-1} g, 1\right)_{D}=\left(g, \frac{1}{1-\bar{\lambda} z}\right)_{D}=(z g)^{\prime}(\lambda) .
$$

This implies that $(z g)^{\prime}$ is analytic in a neighborhood of $\zeta$ for every $g \in$ $\mathscr{M}^{\perp}$. By Theorem 3.2 this means that $\zeta \notin \underline{Z}(\mathscr{M})$.

Now let $\zeta \in \mathbb{T} \backslash \underline{Z}(\mathscr{M})$. Since $\underline{Z}(\mathscr{M}) \cup\{0\}=\underline{Z}(z \mathscr{M})$ and $\varphi \in(z \mathscr{M})^{\perp},(z \varphi)^{\prime}$ extends to be analytic in a neighborhood of $\zeta$. Hence $\varphi$ will be analytic in a simply connected neighborhood of $\zeta$. In [17, Theorem 3.1] it was shown that $\varphi$ must be a multiplier of the Dirichlet space. We leave it to the reader to verify that this implies that the function $\psi(z)=(\varphi(\zeta)-\varphi(z)) /(\zeta-z)$ is a multiplier as well. For $g \in \mathscr{M}^{\perp}$ we have $M_{\varphi}^{*} g=0$, because $\left(M_{\varphi}^{*} g, z^{n}\right)_{D}=\left(g, z^{n} \varphi\right)_{D}=0$ for each $n \geq 0$. Thus

$$
\left(\zeta-M_{z}\right)^{*} M_{\psi}^{*} g=M_{\psi}^{*}\left(\zeta-M_{z}\right)^{*} g=\left(\varphi(\zeta)-M_{\varphi}\right)^{*} g=\overline{\varphi(\zeta) g}
$$

Since $\zeta \notin \underline{Z}(\mathscr{M})=\underline{Z}(\varphi)$ we have $\varphi(\zeta) \neq 0$, so $(1 / \overline{\varphi(\zeta)}) M_{\psi}^{*} \mid \mathscr{M}^{\perp}=$ $\left(\left(\zeta-M_{z}\right)^{*} \mid \mathscr{M}^{\perp}\right)^{-1}$, and $\zeta \in \mathbb{T} \backslash \sigma(T)$.

\section{AN EXAMPLE}

Corollary 3.3 raises the question of exactly which sets can occur as $\underline{Z}(\mathscr{M})$ where $\mathscr{M}$ is an invariant subspace that contains an outer function. As indicated in the remark after the corollary such knowledge would at least in some cases help us decide whether a given function is cyclic or not. In this section we shall show that certain "large" closed sets can occur as $\underline{Z}(\mathscr{M})$.

If an invariant subspace $\mathscr{M}$ contains a nonzero function which extends to be continuous on the closed unit disc, then $\underline{Z}(\mathscr{M}) \cap \mathbb{T}$ has linear Lebesgue measure zero in $\mathbb{T}$. Hence in this case the results on analytic continuation of $\S 3$ imply the existence of the pseudocontinuation (Theorem 2.2). But this is not always the case. In fact, it is well known (see, e.g., [3] or [19]) that there are Blaschke products which divide Dirichlet functions and whose zeros accumulate near every point of the unit circle. Thus there are invariant subspaces $\mathscr{M}$ such that $\underline{Z}(\mathscr{M}) \supseteq \mathbb{T}$, and in particular the functions in $\mathscr{M}$ cannot be continued continuously to $\overline{\mathbb{D}}$.

One might still wonder, whether this can also happen, if the invariant subspace contains an outer function. We shall answer this question affirmatively by constructing an invariant subspace $\mathscr{M}$ which contains an outer function and such that $\underline{Z}(\mathscr{M})=\mathbb{T}$. Thus for such invariant subspaces the results on analytic continuation (Theorem 3.2 and Lemma 3.1(a)) have no content, while Theorem 2.2 still has a strong conclusion.

In our construction we shall make use of a theorem of Carleson's concerning the existence of functions holomorphic in $\mathbb{D}$ and differentiable on $\mathbb{T}$ which vanish on a certain prescribed (necessarily small) subset of $\mathbb{T}$. For this reason we need to recall the notion of a "Carleson thin set". Let $E$ be a compact subset of the unit circle $\mathbb{T}$ and let $\rho_{E}(z)=\operatorname{dist}(z, E)$ for $z \in \mathbb{T}$. E is called a Carleson thin set if $\int_{0}^{2 \pi} \log \rho_{E}\left(e^{i t}\right) d t>-\infty$. It is clear that this condition implies that $|E|=0$, and it is well known that it is equivalent to $|E|=0$ and $\sum_{n=1}^{\infty}\left|I_{n}\right| \log \left(1 /\left|I_{n}\right|\right)<\infty$, where $\left\{I_{n}\right\}$ denotes the collection of complementary 
arcs of $E$, i.e., the $I_{n} \subseteq \mathbb{T}$ are the open disjoint arcs that satisfy $\mathbb{T} \backslash E=\bigcup_{n=1}^{\infty} I_{n}$ (see [2]).

Theorem 4.1 (Carleson [2]). Suppose $E \subseteq \mathbb{T}$ is a Carleson thin set and $n$ is a positive integer. Then there exists an outer function $f \in A$ with $n$ times differentiable boundary function such that $E=Z(f)$.

Lemma 4.2. Let $\varepsilon>0$. There exists a compact set $E \subseteq \mathbb{T}$ and an outer function $\varphi \in D$ having the following properties:

(a) If $I \subseteq \mathbb{T}$ is an open arc such that $E \cap I \neq \varnothing$, then $E \cap I$ has positive logarithmic capacity.

(b) $\|\varphi\|_{\infty} \leq 1$,

(c) $\varphi(0) \geq 1-\varepsilon$,

(d) $\int_{\mathbb{D}}\left|\varphi^{\prime}\right|^{2} d A<\varepsilon^{2}$,

(e) $Z(\varphi)=\underline{Z}(\varphi)=E$.

Proof. First we fix a Carleson thin set $F$ which is contained in $\mathbb{T} \cap\{z: \operatorname{Re} z>0\}$ and which also satisfies condition (a). For example, a Cantor middle thirds set will satisfy these conditions. Next we apply Theorem 4.1 to produce an outer function $f \in A$ such that $f$ is continuously differentiable on $\mathbb{T}$ and $Z(f)=F$. Now choose an outer function $g \in D$ such that $\liminf _{z \rightarrow-1}|g(z)|=\infty$ and such that $|g(z)|$ is continuous on $\overline{\mathbb{D}} \backslash\{-1\}$, e.g., $g(z)=\log \log ((3-z) / 4)$. Then the product $\psi=f g$ is in the Dirichlet space, and by possibly scaling $f$ we may assume that $\int_{\mathbb{D}}\left|\psi^{\prime}\right|^{2} d A<\varepsilon^{2}$. Furthermore, we have $Z(\psi)=\underline{Z}(\psi)=F$, and $\psi$ is unbounded near -1 . Now define an outer function $\psi_{1}$ by $\left|\psi_{1}\left(e^{i t}\right)\right|=$ $\min \left\{1,\left|\psi\left(e^{i t}\right)\right|\right\}$. It follows from our construction that $\left|\psi_{1}(z)\right|$ is continuous on $\overline{\mathbb{D}}$, that $Z\left(\psi_{1}\right)=\underline{Z}\left(\psi_{1}\right)=F$, and that we can choose $\lambda \in \mathbb{D}$ such that $\left|\psi_{1}(\lambda)\right|^{2} \geq 1-\varepsilon$. Also, as remarked earlier the cutoff operation decreases the Dirichlet integral (see, e.g., [17, Lemma 2.6]), thus we have $\int_{\mathbb{D}}\left|\psi_{1}^{\prime}\right|^{2} d A<\varepsilon^{2}$. Finally, we choose a Möbius transformation $\varphi_{\lambda}: \mathbb{D} \rightarrow \mathbb{D}$ which maps 0 to $\lambda$, and set $\varphi(z)=\overline{\psi_{1}(\lambda)} \psi_{1}\left(\varphi_{\lambda}(z)\right)$. It is now clear that $\varphi$ is an outer function which satisfies the conditions (b), (c), (d) of the lemma. Furthermore, condition (e) is satisfied with the set $E=\varphi_{\lambda}^{-1}(F)$. Condition (a) will be satisfied for $E$, because it is satisfied for $F$ and $\varphi_{\lambda}^{-1}$ acts approximately like a dilation on $\mathbb{T}$.

Recall that for $f \in D$ we use $Z(f)$ to denote the radial zero set of $f$, $Z(f)=\left\{z \in \overline{\mathbb{D}}: \lim _{r \rightarrow 1} f(r z)=0\right\}$. If $E \subseteq \mathbb{T}$ is a Borel set of positive logarithmic capacity, then $D_{E}$ is defined to be the invariant subspace

$$
D_{E}=\{f \in D: \log \operatorname{cap} E \backslash Z(f)=0\} \text {. }
$$

Indeed, it is clear that $D_{E}$ is invariant for $\left(M_{z}, D\right)$. That $D_{E}$ is in fact closed is explained in [1, p. 295]. Furthermore, one can show that if $D_{E} \neq(0)$, then it contains nonzero outer functions.

Theorem 4.3. There exists an $F_{\sigma}$ set $E \subseteq \mathbb{T}$ such that $D_{E} \neq(0)$ and $\underline{Z}\left(D_{E}\right)=$ $\mathbb{T}$.

Proof. Choose a sequence $\left\{\varepsilon_{n}\right\}$ of positive numbers such that $\sum \varepsilon_{n}<\infty$. For each $\varepsilon_{n}$ we construct compact sets $E_{n}$ and functions $\varphi_{n}$ as in Lemma 4.2. By rotating the sets $E_{n}$ (and by correspondingly altering $\varphi_{n}$ ) we may assume that the $F_{\sigma}$ set $E=\bigcup_{n>0} E_{n}$ is dense in $\mathbb{T}$. 
Let $\varphi=\prod_{n>0} \varphi_{n}$. It follows easily from our construction that the infinite product converges to a nonzero outer function $\varphi$ in $D$ such that $Z(\varphi) \supseteq E$. Thus $D_{E} \neq(0)$ and $\underline{Z}\left(D_{E}\right) \subseteq \mathbb{T}$.

To show that $\underline{Z}\left(D_{E}\right)=\mathbb{T}$ let $f \in D$ such that $\underline{Z}(f) \neq \mathbb{T}$. We shall show that $f \notin D_{E}$. We can find an open arc $I \subseteq \mathbb{T}$ such that $I \cap \underline{Z}(f)=\varnothing$. But since $E$ is dense in $\mathbb{T}$ there must be an $n$ such that $I \cap E_{n} \neq \varnothing$. By our construction (Lemma 4.2(a)) $I \cap E_{n}$ has positive logarithmic capacity. Of course, we have

$$
I \cap E_{n} \subseteq I \cap E \subseteq E \backslash \underline{Z}(f) \subseteq E \backslash Z(f),
$$

so $E \backslash Z(f)$ has positive logarithmic capacity and $f \notin D_{E}$.

\section{RADIAL LIMITS OF EXTREMAL FUNCTIONS}

In this section we shall prove a few more facts about extremal functions. We shall see that even in all generality and despite the example of the previous section the radial limits of extremal functions have quite regular behavior. In [17, first paragraph of $\S 3$ and Theorem 3.1] it was shown in somewhat greater generality that extremal functions are multipliers and that the multiplier norm of an extremal function is bounded by the maximum of its Hilbert space- and $H^{\infty}$-norms. In the following theorem we shall give a different proof of this fact for the Dirichlet space. This new proof will also show that the $H^{\infty}$-norm of the extremal function is bounded by its Dirichlet norm.

Theorem 5.1. Let $\mathscr{M} \in \operatorname{Lat}\left(M_{z}, D\right), \varphi \in \mathscr{M} \ominus z \mathscr{M}$. Then

(a) $|\varphi(\lambda)| \leq\|\varphi\|_{D} \forall \lambda \in \mathbb{D}$,

(b) $\psi\left(e^{i t}\right)=\lim _{r \rightarrow 1}\left|\varphi\left(r e^{i t}\right)\right|$ exists for every $e^{i t} \in \mathbb{T}$,

(c) $\psi$ is upper semicontinuous on $\mathbb{T}$ and

$$
\limsup _{\lambda \rightarrow e^{i t}, \lambda \in \mathbb{D}}|\varphi(\lambda)|=\psi\left(e^{i t}\right) \quad \forall e^{i t} \in \mathbb{T},
$$

(d) the radial zero set of $\varphi, Z(\varphi)$, equals $\left\{z \in \overline{\mathbb{D}}: \lim _{\sup _{\lambda \rightarrow z}}|\varphi(\lambda)|=0\right\}$ and it is a $G_{\delta}$ set.

It follows from (a) and from the remarks before Theorem 5.1 that the multiplier norm of an extremal function is bounded by its Dirichlet norm.

Proof. It is clear that (c) implies (d). Furthermore, as remarked earlier the Dirichlet space is contained in the space VMO, hence

$$
\lim _{|\lambda| \rightarrow 1}\left(\int_{\mathbb{T}} P_{\lambda}(z)|\varphi(z)|^{2} \frac{|d z|}{2 \pi}-|\varphi(\lambda)|^{2}\right)=0 .
$$

This means that in order to prove (b) and (c) it will be enough to establish statements for the Poisson integral of $|\varphi|^{2}$. We shall do this by use of equation (3.1) of the proof of Lemma 3.1. This formula is valid for all $\lambda=r e^{i t} \in \mathbb{D}$ and it can be written as

$$
\begin{aligned}
\|\varphi\|_{D}^{2} & =\int_{\mathbb{T}} P_{\lambda}(z)\left(|\varphi(z)|^{2}+D_{z}(\varphi)\right) \frac{|d z|}{2 \pi}+r \frac{\partial}{\partial r} \int_{\mathbb{T}} P_{\lambda}(z)|\varphi(z)|^{2} \frac{|d z|}{2 \pi} \\
& =\int_{\mathbb{T}} P_{\lambda}(z) D_{z}(\varphi) \frac{|d z|}{2 \pi}+\frac{\partial}{\partial r} r \int_{\mathbb{T}} P_{\lambda}(z)|\varphi(z)|^{2} \frac{|d z|}{2 \pi} .
\end{aligned}
$$


We integrate this identity in the $r$ variable and obtain for any $r<1$

$$
r\|\varphi\|_{D}^{2}=\int_{0}^{r}\left(\int_{\mathbb{T}} P_{s e^{i t}}(z) D_{z}(\varphi) \frac{|d z|}{2 \pi}\right) d s+r \int_{\mathbb{T}} P_{r e^{i t}}(z)|\varphi(z)|^{\mid} \frac{|d z|}{2 \pi} .
$$

The first integral of the right-hand side is positive and increasing in $r$, hence

$$
|\varphi(\lambda)|^{2} \leq \int_{\mathbb{T}} P_{\lambda}(z)|\varphi(z)|^{2} \frac{|d z|}{2 \pi} \leq\|\varphi\|_{D}^{2},
$$

and $\lim _{r \rightarrow 1} \int_{\mathbb{T}} P_{r e i t}(z)|\varphi(z)|^{2}|d z| / 2 \pi$ exists for every $e^{i t} \in \mathbb{T}$. According to the remark at the beginning of this proof this shows that (a) and (b) are true.

Now let

$$
\psi\left(e^{i t}\right)=\lim _{r \rightarrow 1}\left|\varphi\left(r e^{i t}\right)\right|=\lim _{r \rightarrow 1}\left(\int_{\mathbb{T}} P_{r e^{i t}}(z)|\varphi(z)|^{2} \frac{|d z|}{2 \pi}\right)^{1 / 2} .
$$

To see that (c) is valid as well we notice that in equation (5.1) the first integral is a continuous function of $e^{i t}$. Thus letting $r$ increase to 1 it follows that $\int_{0}^{1}\left(\int_{\mathrm{T}} P_{\text {seit }}(z) D_{z}(\varphi)|d z| / 2 \pi\right) d s$ is a lower semicontinuous function in $e^{i t}$. Hence the function $\psi\left(e^{i t}\right)^{2}$ must be upper semicontinuous in $e^{i t}$. Futhermore, if $L=\limsup _{\lambda \rightarrow e^{i t}}|\varphi(\lambda)|^{2}$, then since $\varphi \in$ VMO we have $L \in$ ess $\operatorname{ran}\left(|\varphi|^{2}, e^{i t}\right)=$ ess $\operatorname{ran}\left(\psi^{2}, e^{i t}\right)$ (here we use again Shapiro's result that for VMO functions the cluster set at the point $e^{i t}$ equals the essential range at $e^{i t}$ [20]), so $L \leq \limsup _{s \rightarrow t} \psi\left(e^{i s}\right)^{2} \leq \psi\left(e^{i t}\right)^{2}$. Of course, the definition of $\psi$ implies that $\psi\left(e^{i t}\right)^{2} \leq L$, so $\lim \sup _{z \rightarrow e^{i t}}|\varphi(z)|^{2}=\psi\left(e^{i t}\right)^{2}$.

\section{A QUESTION}

Theorem 2.2 may be stated in the Bergman space setting. The map $U: D \rightarrow$ $L_{a}^{2}(\mathbb{D}), U g=(z g)^{\prime}$ is unitary, and it is easy to check that the backward Bergman shift $B$,

$$
(B f)(\lambda)=\frac{f(\lambda)-f(0)}{\lambda} \text { for } f \in L_{a}^{2}(\mathbb{D})
$$

satisfies $B U=U\left(M_{z}^{*}, D\right)$, where $\left(M_{z}^{*}, D\right)$ denotes the adjoint of the Dirichlet shift. Thus Theorem 2.2 can be rephrased to say that if a function $h \in L_{a}^{2}(\mathbb{D})$ is not a cyclic vector for $B$, then $h \in N(\mathbb{D})$ and $h$ has a pseudocontinuation $G$ across $\mathbb{T}, G \in N\left(\mathbb{D}^{*}\right)$.

The result of Douglas, Shapiro, and Shields mentioned after Theorem 2.2 characterizes the cyclic vectors of the backward unilateral shift. We had to leave the following problem open:

Problem 6.1. Characterize the cyclic vectors of $B$.

\section{BIBLIOGRAPHY}

1. L. Brown and A. Shields, Cyclic vectors in the Dirichlet space, Trans. Amer. Math. Soc. 285 (1984), 269-304.

2. L. Carleson, Sets of uniqueness for functions regular in the unit circle, Acta. Math. 87 (1952), 325-345.

3. $\ldots$ On the zeros of functions with bounded Dirichlet integrals, Math. Z. 56 (1952), 289-295. 
4. _ـ Selected problems on exceptional sets, Van Nostrand, Princeton, N.J., 1967.

5. J. Douglas, Solution of the problem of Plateau, Trans. Amer. Math. Soc. 33 (1931), 263-321.

6. R. Douglas, H. Shapiro, and A. Shields, Cyclic vectors and invariant subspaces for the backward shift operator, Ann. Inst. Fourier (Grenoble) 20 (1970), 37-76.

7. P. Duren, Theory of $H^{p}$-spaces, Pure and Appl. Math., vol. 38, Academic Press, New York, 1970.

8. J. Garnett, Bounded analytic functions, Pure and Appl. Math., vol. 96, Academic Press, New York, 1981.

9. H. Hedenmalm, A factorization theorem for square area-integrable functions, J. Reine Angew. Math. 422 (1991), 45-68.

10. H. Hedenmalm and A. Shields, Invariant subspaces in Banach spaces of analytic functions, Michigan Math. J. 37 (1990), 91-104.

11. J. Moeller, On the spectra of some translation invariant spaces, J. Math. Anal. Appl. 4(1962), 276-296.

12. S. Richter, Invariant subspaces in Banach spaces of analytic functions, Trans. Amer. Math. Soc. 304 (1987), 585-616.

13. __ Invariant subspaces of the Dirichlet shift, J. Reine Angew. Math. 386 (1988), 205-220.

14. __ A representation theorem for cyclic analytic two-isometries, Trans. Amer. Math. Soc. 328 (1991).

15. S. Richter and A. Shields, Bounded functions in the Dirichlet space, Math. Z. 198 (1988), 151-159.

16. S. Richter and C. Sundberg, A formula for the local Dirichlet integral, Michigan Math. J. 38 (1991), 355-379.

17. __ Multipliers and invariant subspaces of the Dirichlet shift, J. Operator Theory (to appear).

18. H. Shapiro, Some remarks on weighted polynomial approximations of holomorphic functions, Math. Sb. 73(115) (1967), 320-330; English transl. in Math. USSR-Sb. 2 (1967), 285-294.

19. H. Shapiro and A. Shields, On the zeros of functions with finite Dirichlet integral and some related function spaces, Math. Z. 80 (1962), 217-229.

20. J. Shapiro, Cluster set, essential range, and distance estimates in BMO, Michigan Math. J. 34 (1987), 323-336.

21. D. Stegenga, A geometric condition which implies BMOA, Harmonic Analysis in Euclidean Spaces. Proc. Sympos. Pure Math., vol. 35, part 1, Amer. Math. Soc., Providence, R.I., 1979, pp. 427-430.

Department of Mathematics, University of Tennessee, Knoxville, Tennessee 379961300

E-mail address: richter@novell.math.utk.edu

E-mail address: sundberg@novell.math.utk.edu 